\title{
A Hybrid Algorithm of Particle Swarm Optimization and Tabu Search for Distribution Network Reconfiguration
}

\author{
Sidun Fang ${ }^{1,2}$ and Xiaochen Zhang ${ }^{1,2}$ \\ ${ }^{1}$ School of Electronic Information and Electrical Engineering, Shanghai Jiao Tong University, Shanghai 200240, China \\ ${ }^{2}$ Department of Computer Science and Electrical Engineering, Georgia Institute of Technology, Atlanta, GA 30314, USA \\ Correspondence should be addressed to Sidun Fang; fangston@sjtu.edu.cn
}

Received 15 January 2016; Accepted 19 July 2016

Academic Editor: Mauro Pontani

Copyright (c) 2016 S. Fang and X. Zhang. This is an open access article distributed under the Creative Commons Attribution License, which permits unrestricted use, distribution, and reproduction in any medium, provided the original work is properly cited.

\begin{abstract}
This paper deals with the distribution network reconfiguration problem. A hybrid algorithm of particle swarm optimization (PSO) and tabu search (TS) is proposed as the searching algorithm. The new algorithm shares the advantages of PSO and TS, which has a fast computation speed and a strong ability to avoid local optimal solution. After a thorough comparison, network random key (NRK) is introduced as the corresponding coding strategy among various tree representation strategies. NRK could completely avoid the generation of infeasible solutions during the searching process and has a good locality property, which allows the new hybrid algorithm to perform to its fullest potential. The proposed algorithm has been validated through an IEEE 33 bus test case. Compared with other algorithms, the proposed method is both accurate and computationally efficient. Furthermore, a test to solve another problem also proves the robustness of the proposed algorithm for a different problem.
\end{abstract}

\section{Introduction}

Distribution system is usually designed with loops while running in a radial structure. Distribution network reconfiguration (DNR) is a process of altering the topological structure of distribution feeders by changing the open/closed status of the sectionalizing and tie switches [1]. DNR is not limited to fault isolation; from time to time network reconfiguration is performed to achieve various goals, such as system loss reduction, overloads relieving [2], load aggregation [3, 4], and system reliability improvement [5].

The performance and efficiency of any DNR algorithm largely rely on a wise combination of a smart topological coding strategy and an efficient searching algorithm. As a nondeterministic polynomial hard (NP-hard) problem, DNR has been heavily studied with various searching algorithms, from step-by-step heuristics, such as branch-exchange method [6], to metaheuristics based algorithms, such as tabu search (TS) [4], simulated annealing [7], genetic algorithm (GA) [810], and particle swarm optimization (PSO) [11]. Apart from the choice of searching algorithms, the distribution network representation or coding strategy is equally important due to the topological nature of DNR. Various coding strategies have been studied in DNR problem, including binary string representation [5] and Prüfer number representation [3, 12].

After a brief comparison of different coding strategies and existing searching algorithms, this paper proposes a new DNR algorithm. The new method adopts a hybrid optimization of PSO and TS as the searching algorithm and network random keys (NRK) as the corresponding coding strategy. To boost up the overall searching efficiency, a direct method for distribution system power flow analysis [13] is introduced, which has been proved to be both robust and time-efficient.

In recent years, PSO has been successfully applied to solving different kinds of problems, ranging from multimodal and topological mathematical problems $[14,15]$ to aerospace $[11,16-20]$ and chemical engineering $[21,22]$. It is famous for its easy realization and fast convergence, while suffering from the possibility of early convergence to local optimums. In the proposed hybrid algorithm, whenever early convergence occurs, the original particle swarm would be separated into three groups of swarms. Swarm 1 continues performing the basic PSO algorithm; swarm 2 is replaced with newly 
generated random particles; and swarm 3 begins to perform TS on each particle. With the integration of TS, the hybrid PSO algorithm could effectively avoid local optimum by accepting worse solution under certain condition during the searching process.

NRK, which is originally used for GAs, in this paper, has been applied to PSO algorithm. As a topological coding strategy, NRK could completely avoid the possibility of generating unfeasible solutions when using heuristic algorithms in graph optimization problems. It also transforms the original discrete DNR problem into a continuous optimization problem. When applied in GAs, NRK is no more than a coding strategy, which possesses little physical meaning during the solution searching process. However, when used in PSO, the coding strategy has a physical meaning. The value of the "key" in the NRK can be interpreted as an importance index for each branch in the graph. The whole searching process could be interpreted as a process of adjusting the importance index of each branch and choosing the most important branches to form the optimal tree structure.

The remainder of this paper is structured as follows.

In Section 2, the DNR problem is formulated as an optimization problem. In Section 3, different network topology representation schemes are discussed, and NRK is introduced. In Section 4, the hybrid algorithm of PSO and TS is proposed and explained in detail. In Section 5, the new algorithm is tested on an IEEE 33 bus system with numerical results. The conclusion is drawn in Section 6.

\section{Problem Representation}

DNR is originally used in planned outages for maintenance purpose or fault isolation to restore service. A. Merlin and $\mathrm{H}$. Back [14] were the first to come to the idea that reconfiguration may lead to a system total loss reduction and they tried to search for such an optimal configuration using the branch-and-bound method. Since then, loss reduction has been considered as a common objective for the study of new DNR optimization algorithms.

A noticeable characteristic of DNR is the repeated analysis of power flow during the solution searching process. In order to improve searching efficiency, several refined or approximate algorithms for power flow analysis have been studied, such as decoupled method [15], hashing table method [16], and perturbation method [2]. A direct method [13] is adopted in this paper, which has been proved to be highly efficient in distribution network power flow analysis.

Another characteristic of DNR is the topological constraints, which means any feasible solution of a DNR problem should represent a tree structure with every node being connected. Configuration space is the set of allowed system configurations over which the optimal system configuration is to be searched for [7]. In DNR, only solutions that belong to the configuration space are considered feasible.

Assume that a distribution network has $n$ branches. A DNR problem for system losses reduction can be formulated as

$$
\min f=\sum_{b=1}^{n} x_{b} r_{b} \frac{P_{b}^{2}+Q_{b}^{2}}{V_{b}^{2}}+\alpha P\left(V_{b}\right)+\beta P\left(I_{b}\right),
$$

where $x$ is an $n$-dimensional vector. If branch $b$ is closed, $x_{b}=1$; otherwise $x_{b}=0 . P_{b}$ and $Q_{b}$ represent the active and reactive power flow on branch $b . \alpha$ and $\beta$ are penalty factors, while $P\left(V_{b}\right)$ and $P\left(I_{b}\right)$ are penalty functions for node voltage constraint and branch current constraint.

\section{Network Topology Representation}

The process of searching the optimal DNR solution involves the graph theory of optimal spanning tree. Let graph $G(V, E)$ represent the topology of a distribution network, where $V$ stands for vertices and $E$ stands for edges. Each potential solution is a spanning tree of $G$. All spanning trees of the graph $G$ make up the configuration space. A good network topology representation strategy should have four characteristics:

(1) Being Easy to Encode and Decode. A less complicated coding strategy would cost less time to encode and decode, thus leading to a boost in computational efficiency.

(2) Being Compatible with Other Optimization Algorithms. Many metaheuristic algorithms have their own limitations in dealing with different types of optimization problems. For example, GAs require a binary string representation, and PSO requires continuous variables. A good coding strategy should be compatible with corresponding searching algorithms.

(3) Avoiding Infeasible Solutions. Topological constraint is one of the thorniest issues in DNR, especially when it comes to the utilization of metaheuristic algorithms. Whenever an infeasible solution is generated, the original searching process will be interrupted. A good coding strategy should effectively rule out the possibility of generating infeasible solutions, which would greatly improve the computational efficiency and avoid the tedious topological checking process.

(4) Having a Good Locality Property. A good locality property means that the objective function value is relatively continuous and smooth, rather than irregular jumps within a local area in the searching space. Most metaheuristic algorithms determine the best searching direction based on current objective function values. Then the algorithms will lead the searching process towards the most promising direction. In other words, a coding strategy with bad locality properties will greatly restrict the effectiveness of the searching algorithm. The configuration space generated by a good coding strategy should always keep a high locality.

There are many different ways to represent the distribution network topology, and each of them has its merits and flaws.

Binary string representation is the most intuitive and straightforward way to represent the network topology by assigning a binary string $x$. The dimension of $x$ is the total number of switches. The elements in $x$ are set to be 0 or 
1, representing the open and closed status of each switch. However, binary string representation is usually blamed for the high probability of generating infeasible solutions when applied by many searching algorithms such as SA or PSO. Genetic operators such as crossover or mutation almost always generate infeasible solution, which forces the algorithm to stop.

In order to reduce the probability of generating infeasible solutions, homeomorphism [12] and fundamental loop [17] representation method are widely adopted. The graph theory of homeomorphism simplifies the original graph by smoothing out unnecessary vertices from the original graph. After the simplification, each branch in the new graph represents a group of branches in the original graph. According to the graph theory, one and only one branch could be opened in each branch group in order to form a tree structure. Similarly, fundamental loop representation avoids infeasible solutions by introducing fundamental loop tables. Only one branch should be opened in each fundamental loop. These two methods help to reduce the probability of generating infeasible solutions and keep the searching process from interruption. However, none of the methods above could completely avoid infeasible solutions, and additional checking rules are still necessary.

Random key (RK) is an efficient method for encoding and scheduling problems. Rothlauf et al. [9] proposes a tree representation for GAs using RK, by the name of network random keys. Queiroz and Lyra [3] are the first to introduce the combination of NRK and GAs in the DNR problem.

Taking a 5-node system as an example, see Figure 1. The NRK coding and decoding process goes as follows.

Step 1. Generate a "key" vector with the dimension of 6 , denoted as $x$. And each element in $x$ stands for a branch in the network. The value of each key is a random number $\in[0,1]$.

Step 2. Rank the elements in "key" vector according to their value in descending order, denoted as $x^{\prime}$.

Step 3. Let $F$ stand for a branch set which contains the branches chosen to form the tree. Starting from the first element in $x^{\prime}$, add one branch into $F$ at a time. Whenever a loop is formed, abandon the latest added branch and continue adding branches with the next element, until all 4 branches have been chosen.

The ordering of the branches in $x^{\prime}$ would change according to the variation of values in the "key" vector. Consequently, the final tree structure would also change. Branch with a higher "key" value will rank in the front and is more likely to be chosen to form the final tree structure. Similarly, branches with lower "key" values are more unlikely to be chosen. In other words, a higher "key" value means a higher importance of that branch.

To begin with, as a tree representation strategy, NRK could guarantee that any "key" vector could generate one and only one feasible solution, making the topological checking process completely unnecessary. Moreover, by using RKs, which are continuous variables, NRK transforms the original discrete DNR problem into a continuous problem. As a result, algorithms such as basic PSO could be applied to NRK directly. Finally, since the construction of the tree is based on the relative order of the branches, the locality of NRK is very high, which is a good prerequisite for other optimization algorithms to perform to their fullest potential.

\section{Hybrid Algorithm}

Various algorithms have been implemented in DNR problem, including GAs, TS, and SA. In this paper, a hybrid algorithm of PSO and TS has been introduced. The new algorithm shares the advantages of both PSO and TS.

Tabu search is a local search algorithm that can be used for solving combinatorial optimization problems. It uses some memory structures such as tabu list or frequency list to force the searching process to cover new searching area and prevent early convergence to the local optimal solution. The advantage of TS lies in a strong local searching ability and the ability to jump out of local optimum. In [4], tabu search is successfully applied to DNR with some necessary modifications. Meanwhile, the disadvantage of TS is its strong dependence on a proper initial solution and relatively low searching efficiency compared with other metaheuristics.

PSO is a stochastic optimization technique developed by Kennedy and Eberhart [23]. The algorithm introduces a number of particles to form a swarm. Each particle travels in the searching space to search for the global optimum using the experiences of other particles [16]. It has the features of parallel computing and high computational efficiency. However, PSO also suffers from the probability of early convergence. With improper parameters, PSO may easily fall into local optimum. Since the original PSO is designed for continuous variable optimization, binary particle swarm optimization (BPSO), a modified PSO algorithm, is introduced to deal with DNR problem [11].

In the basic PSO algorithm, particle updates its velocity and position with the following equations:

$$
\begin{aligned}
& v_{i d}=v_{i d}+c_{1} r_{1}\left(p_{i d}-x_{i d}\right)+c_{2} r_{2}\left(p_{g d}-x_{i d}\right), \\
& x_{i d}=x_{i d}+v_{i d},
\end{aligned}
$$

where $x_{i d}$ and $v_{i d}$ stand for the position and velocity of the $d$ th dimension of particle $i$; $p_{i d}$ and $p_{g d}$ stand for the particle best and global best position; $c_{1}$ and $c_{2}$ are nonnegative constants; and $r_{1}$ and $r_{2}$ are two random numbers $\in[0,1]$.

In order to overcome the various shortcomings of PSO and TS, a hybrid algorithm is proposed. On the one hand, PSO could greatly increase the searching efficiency; on the other hand, TS would help to avoid local optimal solution. Moreover, TS's strong local searching ability may even increase PSO's accuracy when the true global optimum is not far away. The flow chart of the new algorithm is shown in Figure 2. In the hybrid algorithm, the original particle swarms are partitioned into three swarms: swarm 1, swarm 2, and swarm 3. At the beginning, the new algorithm performs just as the basic PSO algorithm. When the algorithm stopped updating $p_{i d}$ and $p_{g d}$ within certain period of time, which might be a sign of early convergence, the new algorithm 


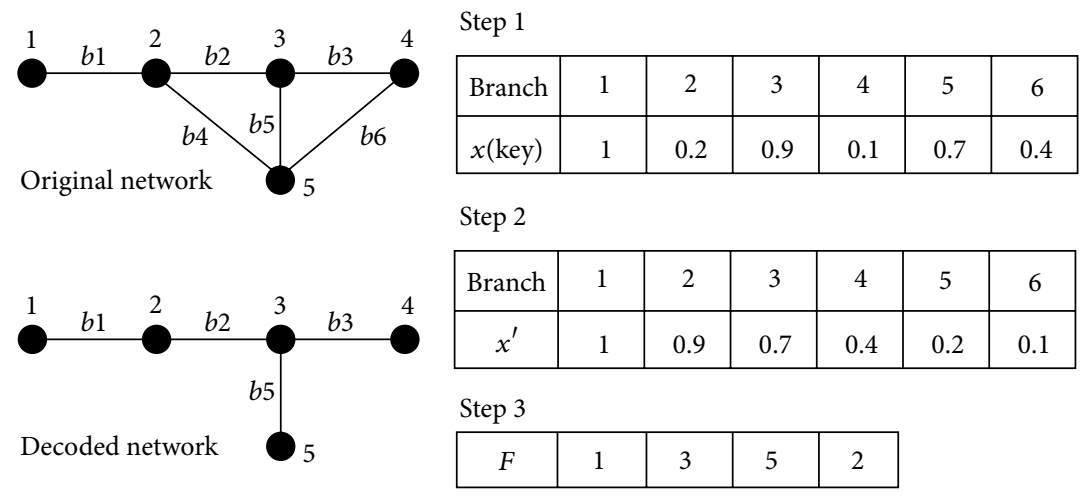

FIGURE 1: NRK coding and decoding process.

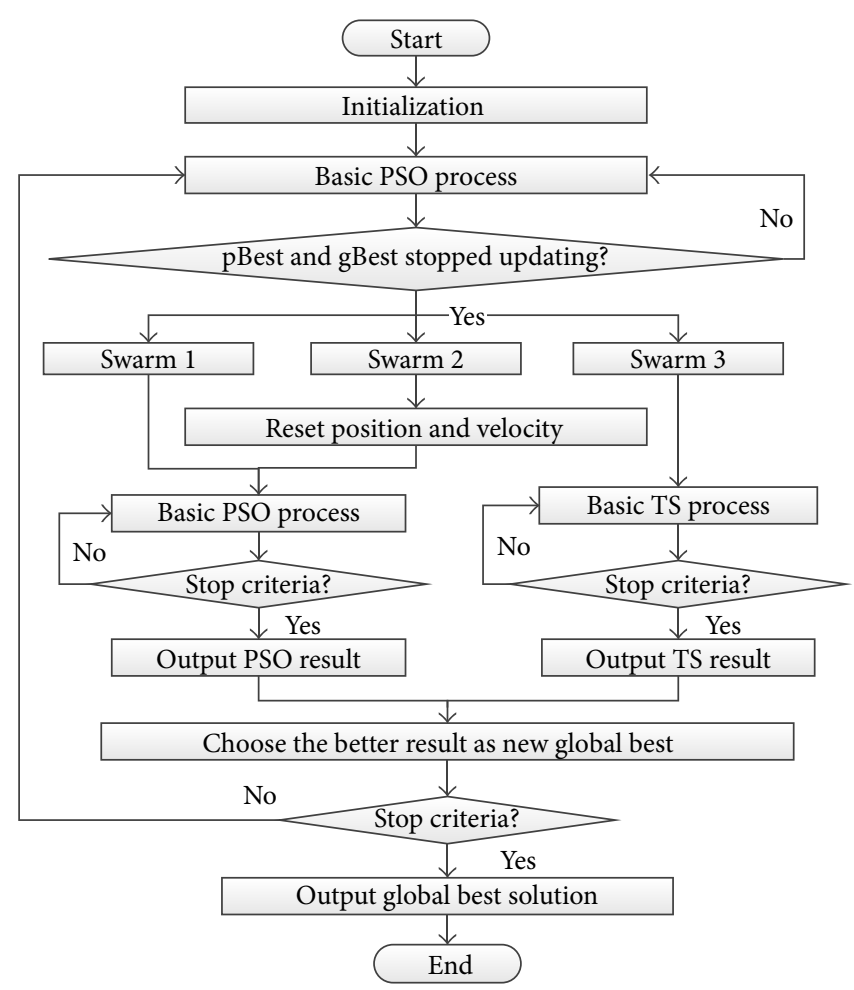

FIGURE 2: Hybrid algorithm flow chart.

would begin to perform differently. Swarm 1 would continue performing basic PSO algorithm using the equation above. Swarm 2 would be reset with new random positions and velocities. Swarm 3 would perform TS based on the current optimal solution.

\section{Case Studies}

The proposed hybrid algorithm was realized using MATLAB and tested on the IEEE 33-node system [2]; see Figure 3. The system consists of 37 branches and 33 nodes. The number of fundamental loops is $37-33+1=5$. The original system losses are $202.67 \mathrm{~kW}$, with branches $33,34,35,36$, and 37 opened. Under the optimal network configuration, the system losses should be $128.72 \mathrm{~kW}$, with branches 7, 9, 14, 31, and 37 opened.

In order to compare the performances between the proposed hybrid algorithm and other basic algorithms, three difference cases are studied.

In case 1 , binary string representation is adopted as the tree representation method. BPSO is performed to deal with the discrete optimization problem. The logical transformation function of BPSO is established as the sigmoid function in [18]. Both the BPSO parameters $c_{1}$ and $c_{2}$ are set to be 4 . As binary string representation allows the generation of infeasible solutions, whenever an infeasible solution is 


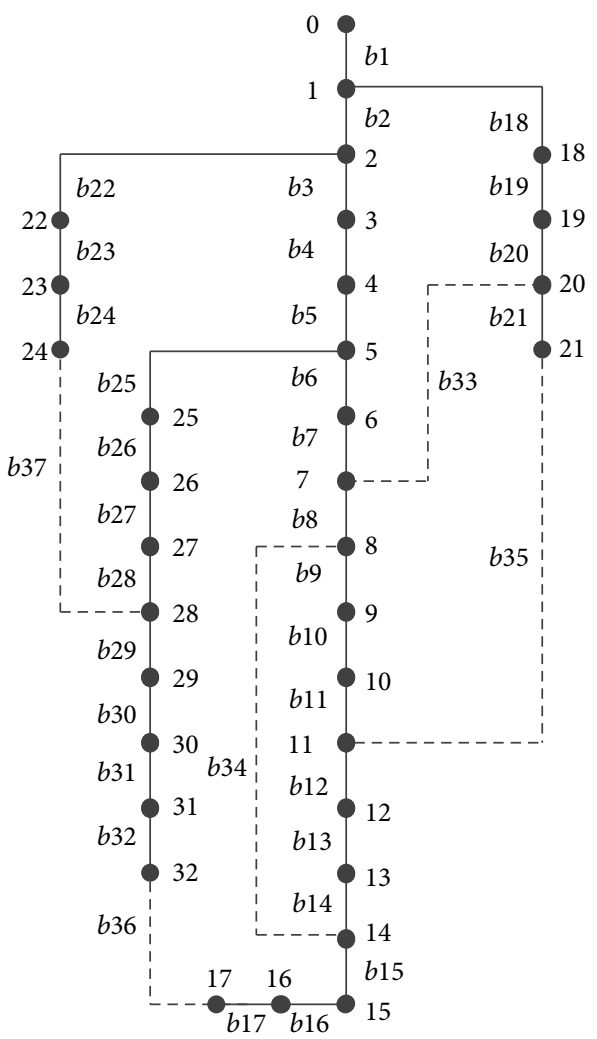

FIgURE 3: IEEE 33 bus case.

TABLE 1: System losses and computation time.

\begin{tabular}{lccc}
\hline Case ID & \multicolumn{2}{c}{ Optimal configuration (kW) } & $\begin{array}{c}\text { Mean computation } \\
\text { time (seconds) }\end{array}$ \\
\hline Case 1 & 135.03 & 2.1 & 29.36 \\
Case 2 & 134.19 & 2.91 & 16.64 \\
Case 3 & 130.33 & 2.23 & 17.12 \\
\hline
\end{tabular}

TABLE 2: Histogram of system losses.

\begin{tabular}{lccc}
\hline \multirow{2}{*}{ Case ID } & \multicolumn{3}{c}{ Percentage among 100 trials } \\
& Losses $=128.72$ & Losses $<134.00$ & Losses $<139.00$ \\
\hline Case 1 & $2 \%$ & $11 \%$ & $69 \%$ \\
Case 2 & $26 \%$ & $41 \%$ & $90 \%$ \\
Case 3 & $89 \%$ & $86 \%$ & $97 \%$ \\
\hline
\end{tabular}

The system losses for the true optimal solution are $128.72 \mathrm{~kW}$.

TABLE 3: The results of PSO.

\begin{tabular}{lcccc}
\hline \multirow{2}{*}{ Parameter } & \multicolumn{4}{c}{ Percentage among 100 trials } \\
& $c_{2}=2$ & $c_{2}=3$ & $c_{2}=4$ & $c_{2}=5$ \\
\hline$c_{1}=2$ & $17 \%$ & $16 \%$ & $18 \%$ & $20 \%$ \\
$c_{1}=3$ & $19 \%$ & $22 \%$ & $22 \%$ & $23 \%$ \\
$c_{1}=4$ & $20 \%$ & $23 \%$ & $26 \%$ & $25 \%$ \\
$c_{1}=5$ & $21 \%$ & $23 \%$ & $26 \%$ & $26 \%$ \\
\hline
\end{tabular}

Table 2 shows the percentage of solutions with a system generated, a feasible particle is picked to replace the infeasible one.

In case 2, NRK is chosen as the tree representation method. As continuous variables, "keys" enable basic PSO algorithm to perform directly into DNR problem. The basic PSO parameters are set as follows: $c_{1}=c_{2}=4$. Each "key" value in NRK vector is limited within $[0,1]$.

In case 3 , the new hybrid algorithm of PSO and TS is adopted, with NRK being the tree representation method. The PSO parameters are the same as case 2 . The length of tabu list is 3 ; the length of frequency list is 10 . The frequency penalty factor is set to be 1.2. The detailed aspiration criterion is explained in [19].

To get a population large enough to study the differences among the three cases, each case is performed 100 times. The particle numbers in all cases are set to 10 , and the maximum iteration time is set as 50. The average computation time and losses achieved by three cases in 100 trails are shown in Table 1. The standard deviation (STDV) of losses enables evaluating the algorithms' robustness.

By comparing case 1 with case 2 , we can see that the bad locality property of binary string greatly limited the performance of PSO and the high probability of generating of infeasible particles significantly slowed the entire searching process. By comparing case 2 with case 3 , we can see that the integration of tabu search could effectively increase the local search ability and help PSO jump out of local optimal solution.

loss less than certain criteria within 100 trials for each case. It further illustrates that both NRK and the hybrid algorithm significantly increase the probability of finding the global optimal solution. 69 out of 100 trails have found the global optimal solution in the new algorithm, which is much higher than other two cases.

Figure 4 shows the global best solution curve during 50 iterations for all cases. In the hybrid algorithm, when pBest and gBest stopped updating for a period of time, a local optimal solution is found, as seen in Figure 4: "Hybrid-PSO." Then the original swarm process is forced to stop at the 41th iteration. Then the original particle swarm is separated into three swarms. "Hybrid-TS" shows how tabu search help a particle in swarm 3 jump out of the local optimum and increase the possibility of finding the global optimal network configuration.

To test the parametric robustness of the proposed algorithm, the percentages to get global best among 100 trials of PSO and hybrid algorithm under different parameter combinations are shown in Tables 3 and 4, respectively.

From the results of Tables 3 and 4, the hybrid algorithm has similar parameter selection region compared to the PSO. The best parameters $\left(c_{1}\right.$ and $\left.c_{2}\right)$ of two algorithms are both 4 or 5. That means the hybrid process has no evident impacts on the parameter selection. The main reason is that the PSO process and TS process are independent of each other. The TS process is activated when the solving process is caught into 


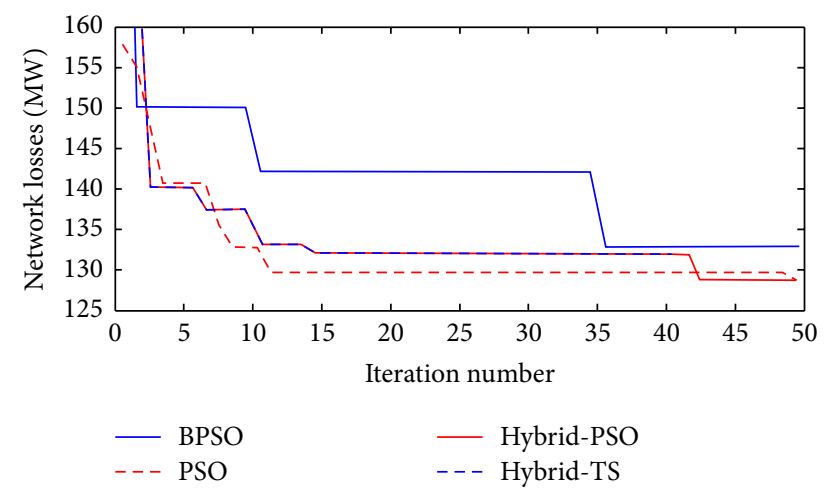

FIGURE 4: Global best solution during the searching process.

TABLE 4: The results of proposed method.

\begin{tabular}{lcccc}
\hline \multirow{2}{*}{ Parameter } & \multicolumn{4}{c}{ Percentage among 100 trials } \\
& $c_{2}=2$ & $c_{2}=3$ & $c_{2}=4$ & $c_{2}=5$ \\
\hline$c_{1}=2$ & $73 \%$ & $75 \%$ & $79 \%$ & $82 \%$ \\
$c_{1}=3$ & $78 \%$ & $80 \%$ & $83 \%$ & $85 \%$ \\
$c_{1}=4$ & $80 \%$ & $85 \%$ & $89 \%$ & $89 \%$ \\
$c_{1}=5$ & $81 \%$ & $87 \%$ & $90 \%$ & $91 \%$ \\
\hline
\end{tabular}

TABLE 5: The comparison between PSO and proposed hybrid algorithm.

\begin{tabular}{lccc}
\hline \multirow{2}{*}{ Method } & \multicolumn{3}{c}{ Percentage among 100 trials } \\
& Losses $=112.16$ & Losses $<115$ & Losses $<118$ \\
\hline PSO & $5 \%$ & $14 \%$ & $55 \%$ \\
Hybrid & $31 \%$ & $67 \%$ & $100 \%$ \\
\hline
\end{tabular}

local best. Thus the parameter tuning problems do not exist in the proposed hybrid process.

To prove the robustness of the proposed hybrid algorithm to different problems, PSO and the proposed hybrid algorithm are both tested in a reactive power dispatch model [24]. The test system is the IEEE 118 bus system. The algorithmic parameters are the same with case 1 and case 2 . The results are shown in Table 5.

From Table 5, the proposed hybrid algorithm also has superior characteristics compared to PSO, which shows that proposed method is able to enhance the global searching ability of PSO for different models.

\section{Conclusion}

PSO is a very promising algorithm to large scale optimization problems as DNR problem. The main contribution of this paper is presenting a hybrid PSO searching algorithm and introduces NRK as the tree representation strategy for the new algorithm.
The numerical results drawn from the test system validate the effectiveness and efficiency of both the hybrid algorithm and the introduction of the new coding strategy.

The main drawback of the algorithm lies in the situation that PSO algorithm converges to a local optimal solution which is located very far away from the true global optimal solution; tabu search may not have the ability to jump out of such a very deep local optimal solution. Another drawback is that a subtle change of "key" value does not necessarily lead to a change in the tree structure formulation, unless the change is big enough to change the importance sequence of the branches. In other words, NRK is not very sensitive to PSO algorithm, and that is also the very reason why algorithms with strong local search ability like tabu search are indispensable.

Further analysis shows that the hybrid process has no evident influences on the parameter selection, and the proposed method is also able to enhance the global searching ability of PSO in different problems.

\section{Competing Interests}

The authors declare that they have no competing interests.

\section{References}

[1] S. Civanlar, J. J. Grainger, H. Yin, and S. S. H. Lee, "Distribution feeder reconfiguration for loss reduction," IEEE Transactions on Power Delivery, vol. 3, no. 3, pp. 1217-1223, 1988.

[2] M. E. Baran and F. F. Wu, "Network reconfiguration in distribution systems for loss reduction and load balancing," IEEE Transactions on Power Delivery, vol. 4, no. 2, pp. 1401-1407, 1992.

[3] L. M. O. Queiroz and C. Lyra, "Adaptive hybrid genetic algorithm for technical loss reduction in distribution networks under variable demands," IEEE Transactions on Power Systems, vol. 24, no. 1, pp. 445-453, 2009.

[4] S. F. Mekhamer, A. Y. Abdelaziz, F. M. Mohammed, and M. A. L. Badr, "A new intelligent optimization technique for distribution systems reconfiguration," in Proceedings of the 2008 12th International Middle East Power System Conference (MEPCON '08), pp. 397-401, IEEE, Aswan, Egypt, March 2008.

[5] B. Amanulla, S. Chakrabarti, and S. N. Singh, "Reconfiguration of power distribution systems considering reliability and power loss," IEEE Transactions on Power Delivery, vol. 27, no. 2, pp. 918-926, 2012.

[6] D. W. Ross, M. Carson, and A. I. Cohen, "Development of advanced method for planning electric energy distribution systems," U.S.DOE Report ET-8-c-03-1845, 1980.

[7] H. D. Chiang and R. Jean-Jumeau, "Optimal network reconfigurations in distribution systems. II. Solution algorithms and numerical results," IEEE Transactions on Power Delivery, vol. 5, no. 3, pp. 1568-1574, 1990.

[8] K. Nara, A. Shiose, M. Kitagawa, and T. Ishihara, "Implementation of genetic algorithm for distribution systems loss minimum re-configuration," IEEE Transactions on Power Systems, vol. 7, no. 3, pp. 1044-1051, 1992.

[9] F. Rothlauf, D. E. Goldberg, and A. Heinzl, "Network random keys-a tree representation scheme for genetic and evolutionary algorithms," Evolutionary Computation, vol. 10, no. 1, pp. 7597, 2002. 
[10] E. M. Carreno, N. Moreira, and R. Romero, "Distribution network reconfiguration using an efficient evolutionary algorithm," in Proceedings of the IEEE Power Engineering Society General Meeting (PES '07), pp. 1-6, Tampa, Fla, USA, June 2007.

[11] P. C. Fourie and A. A. Groenwold, "The particle swarm optimization algorithm in size and shape optimization," Structural and Multidisciplinary Optimization, vol. 23, no. 4, pp. 259-267, 2002.

[12] J. Kennedy and R. C. Eberhart, "Discrete binary version of the particle swarm algorithm," in Proceedings of the 1997 IEEE International Conference on Systems, Man, and Cybernetics. Part 1 (of 5), pp. 4104-4108, October 1997.

[13] G. R. Raidl and B. A. Julstrom, "Edge sets: an effective evolutionary coding of spanning trees," IEEE Transactions on Evolutionary Computation, vol. 7, no. 3, pp. 225-239, 2003.

[14] K. Parsopoulos and M. N. Vrahatis, "On the computation of all global minimizers through particle swarm optimization," IEEE Transactions on Evolutionary Computation, vol. 8, no. 3, pp. 211224, 2004.

[15] P. C. Fourie and A. A. Groenwold, "Particle swarms in topology optimization," in Proceedings of the 4th World Congress of Structural and Multidisciplinary Optimization, pp. 1771-1776, Liaoning Electronic Press, 2001.

[16] M. Pontani, C. Martin, and B. A. Conway, "New numerical methods for determining periodic orbits in the circular restricted three-body problem," in Proceedings of the 61st International Astronautical Congress (IAC '10), pp. 499-509, Prague, Czech Republic, October 2010.

[17] M. Pontani and B. A. Conway, "Particle swarm optimization applied to space trajectories," Journal of Guidance, Control, and Dynamics, vol. 33, no. 5, pp. 1429-1441, 2010.

[18] C. R. Bessette and D. B. Spencer, "Optimal space trajectory design: a heuristic-based approach," in Proceedings of the Spaceflight Mechanics 2006-AAS/AIAA Space Flight Mechnaics Meeting, vol. 124 of AAS paper 06-197, pp. 1611-1628, San Diego, Calif, USA, January 2006.

[19] K.-J. Zhu, J.-F. Li, and H.-X. Baoyin, "Satellite scheduling considering maximum observation coverage time and minimum orbital transfer fuel cost," Acta Astronautica, vol. 66, no. 1-2, pp. 220-229, 2010.

[20] K. Zhu, F. Jiang, J. Li, and H. Baoyin, "Trajectory optimization of multi-asteroids exploration with low thrust," Transactions of the Japan Society for Aeronautical and Space Sciences, vol. 52, no. 175, pp. 47-54, 2009.

[21] A. R. Cockshott and G. R. Sullivan, "Improving the fermentation medium for Echinocandin B production part II: particle swarm optimization," Process Biochemistry, vol. 36, no. 7, pp. 661-669, 2001.

[22] C. O. Ourique, E. C. Biscaia Jr., and J. C. Pinto, "The use of particle swarm optimization for dynamical analysis in chemical processes," Computers \& Chemical Engineering, vol. 26, no. 12, pp. 1783-1793, 2002.

[23] J. Kennedy and R. Eberhart, "Particle swarm optimization," in Proceedings of the IEEE International Conference on Neural Networks, pp. 1942-1948, Perth, Australia, December 1995.

[24] S. Fang, H. Cheng, Y. Song et al., "Stochastic optimal reactive power dispatch method based on point estimation considering load margin," in Proceedings of the IEEE PES General MeetingConference \& Exposition, pp. 1-5, National Harbor, Md, USA, July 2014. 


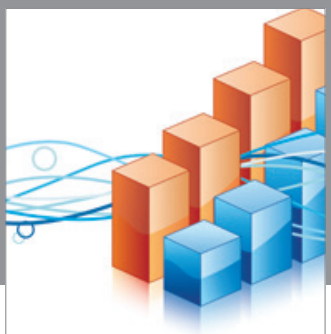

Advances in

Operations Research

vatem alat4

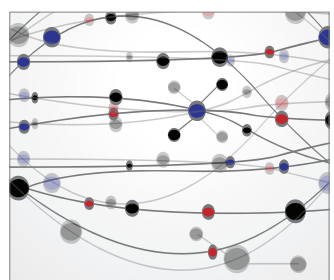

\section{The Scientific} World Journal
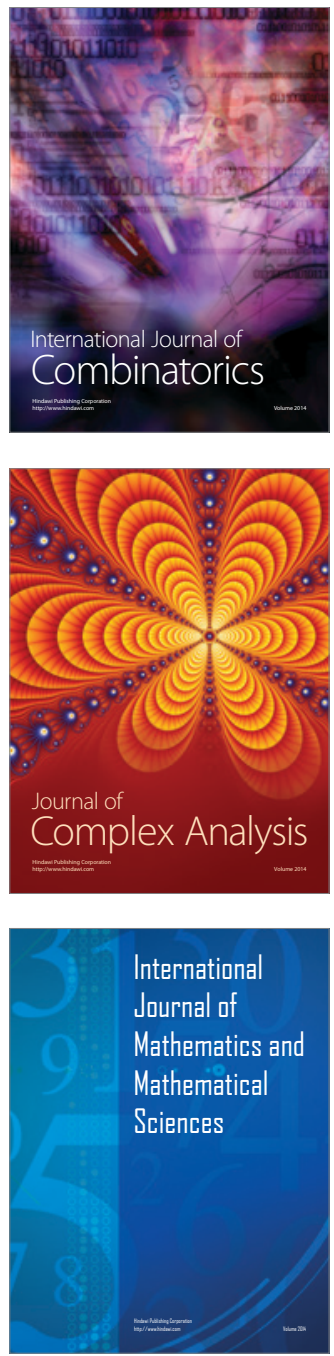
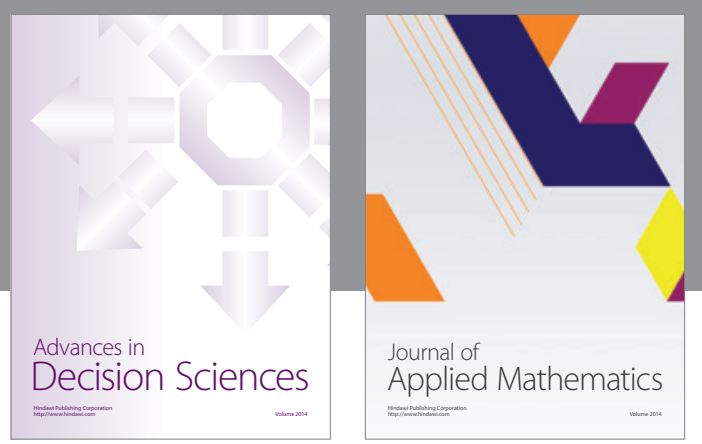

Algebra

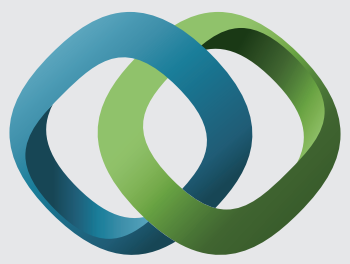

\section{Hindawi}

Submit your manuscripts at

http://www.hindawi.com
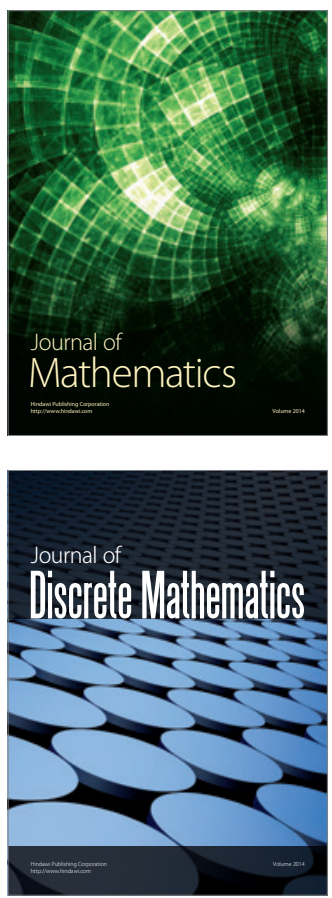

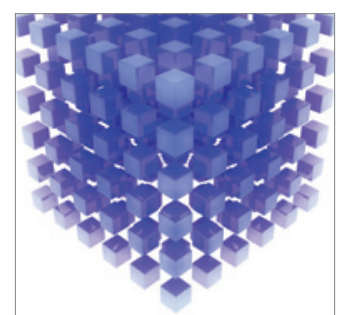

Mathematical Problems in Engineering
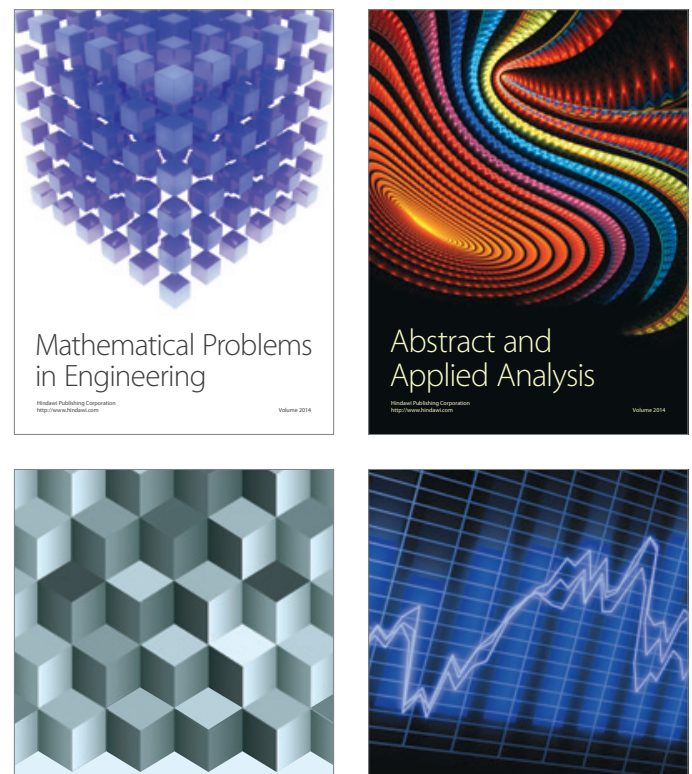

Journal of

Function Spaces

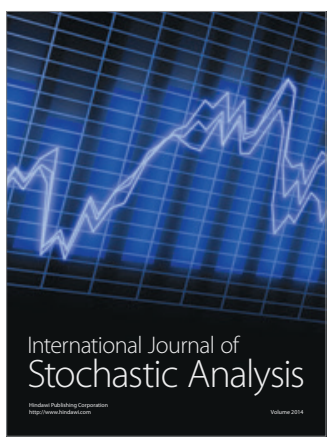

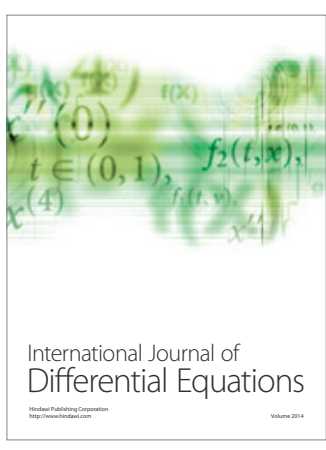
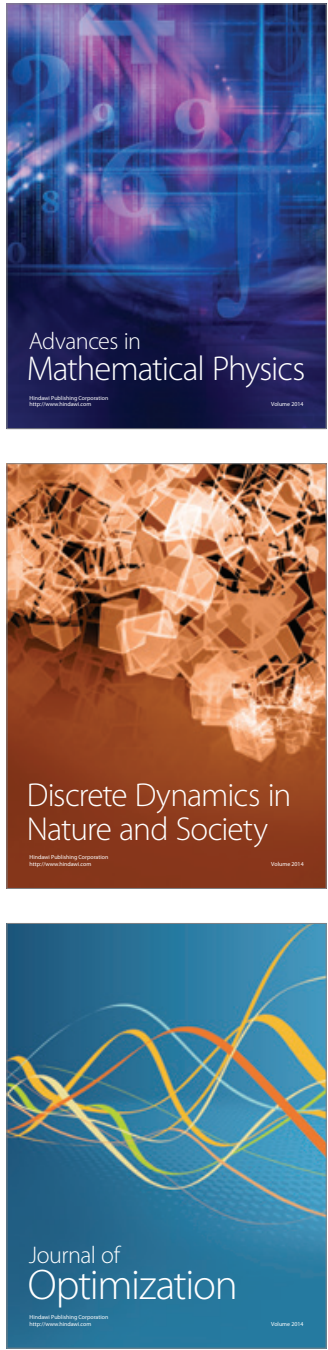